\title{
The therapeutic promise of the cancer stem cell concept
}

\author{
Natasha Y. Frank, ${ }^{1,2}$ Tobias Schatton, ${ }^{3}$ and Markus H. Frank ${ }^{3,4}$
}

1Division of Genetics, Brigham and Women's Hospital, Boston, Massachusetts, USA. 2Department of Medicine, VA Boston Healthcare System, West Roxbury, Massachusetts, USA. ${ }^{3}$ Transplantation Research Center, Children's Hospital Boston, and Brigham and Women's Hospital, Harvard Medical School, Boston, Massachusetts, USA. “Department of Dermatology, Brigham and Women's Hospital, Harvard Medical School, Boston, Massachusetts, USA.

\begin{abstract}
Cancer stem cells (CSCs) are a subpopulation of tumor cells that selectively possess tumor initiation and self-renewal capacity and the ability to give rise to bulk populations of nontumorigenic cancer cell progeny through differentiation. As we discuss here, they have been prospectively identified in several human malignancies, and their relative abundance in clinical cancer specimens has been correlated with malignant disease progression in human patients. Furthermore, recent findings suggest that clinical cancer progression driven by CSCs may contribute to the failure of existing therapies to consistently eradicate malignant tumors. Therefore, CSC-directed therapeutic approaches might represent translationally relevant strategies to improve clinical cancer therapy, in particular for those malignancies that are currently refractory to conventional anticancer agents directed predominantly at tumor bulk populations.
\end{abstract}

\section{Cancer stem cells: definition and experimental identification}

Physiological tissues are hierarchically organized, that is, they are composed of cell populations with diverse self-renewal and proliferative capacities. Relatively rare, uncommitted, quiescent tissue-specific stem cells are found at the apices of these cellular hierarchies and are defined by 2 distinct properties: the capacity for prolonged self-renewal and the potential to give rise to more mature, transiently amplifying cell progenies that in turn give rise to specialized cells of particular tissues through differentiation (1). In addition, such stem cells possess the capacity to proliferate extensively (1), for example, in response to injury and during development (2). Bidirectional interactions between these stem cells and the cellular constituents of their individual niches involve distinct developmental signaling networks, soluble mediators, and/or cellmatrix processes. These interactions are essential for the establishment of a stem cell-permissive microenvironment and provide a crucial regulatory balance between self-renewal and differentiation and between quiescence and proliferation (3).

Some malignant tumors can also be composed of morphologically and phenotypically heterogeneous cell populations $(4,5)$ with varying self-renewal capacities, degrees of differentiation, and clonogenic and tumorigenic potentials (6-10). Moreover, many of the signaling cascades and interactions with stromal elements that orchestrate physiological stem cell behavior, and consequently normal development, have also been found to play important roles in the initiation and progression of tumors (11). Taken together, these observations have led to the development of the cancer stem cell (CSC) theory, which posits that neoplasms, like physiologic tissues, can be hierarchically organized, and that CSCs, which are found at the apex of this cellular hierarchy and seem to comprise only a subpopulation of tumor cells, are essential for its propagation (1). According to a consensus definition (12), a CSC is a cell within a tumor that possesses the capacity to self-renew and to generate the heterogeneous lineages of cancer cells that comprise the tumor. Therefore, CSCs can only be defined experimentally by their ability to recapitulate the generation of a

Conflict of interest: The authors have declared that no conflict of interest exists. Citation for this article: J. Clin. Invest. 120:41-50 (2010). doi:10.1172/JCI41004. continuously growing tumor (12). Consensus also exists that the gold standard assay that fulfills these criteria is serial transplantation in animal models, which, although imperfect, is regarded as the best functional assay for the 2 critical criteria of the consensus CSC definition (12). Clearly, as discussed previously (13), tumorigenicity in human-to-mouse xenotransplantation models, and as a result calculated CSC frequency estimates, might vary with the applied experimental conditions, such as the tissue site of xenotransplantation and the presence or absence of immune effector mechanisms in recipient immunodeficient mice. The dependence of tumorigenic potential on the immune status of the tumor host has been confirmed in human malignant melanoma xenograft models (14). However, this study did not directly address CSCspecific functions such as self-renewal and differentiation capacity in marker-tracked serial xenotransplantation experiments (14). Microenvironmental factors can also markedly influence cancer cell tumorigenicity in xenotransplantation models (15), as shown for human melanoma, in which exogenously added ECM factors normally produced by tumor cells markedly enhanced tumorigenic potential (14), and for human breast cancer, in which cografted cancer-derived fibroblasts substantially enhanced tumor growth in immunodeficient nude mice due to increased production of stromal-derived factor-1 and resultant paracrine stimulation of breast cancer CXCR4 (16).

The diversity and complexity of currently available experimental tumor xenotransplantation models, and the distinct results that have been generated in each particular assay, suggest that there might not exist a single ideal or best-suited model for the study of CSCs, but rather that cumulative knowledge generated in the aggregate of existing and potential future models will yield increasingly important and definitive insights into CSC biology.

Although CSCs have been thought to comprise a relatively rare subpopulation of tumor cells in several malignancies, relative rarity is not a defining criterion according to the consensus CSC definition (12) and is not necessarily a defining feature in all cancers in which CSCs have been identified $(13-15,17,18)$. In addition, it is noteworthy that the CSC concept does not make any specific assumptions about the multipotent transdifferentiation plasticity of the CSC subset $(12,18)$ or about the cell from which the cancer arose (12), that is, it does not address whether the cancer arose 

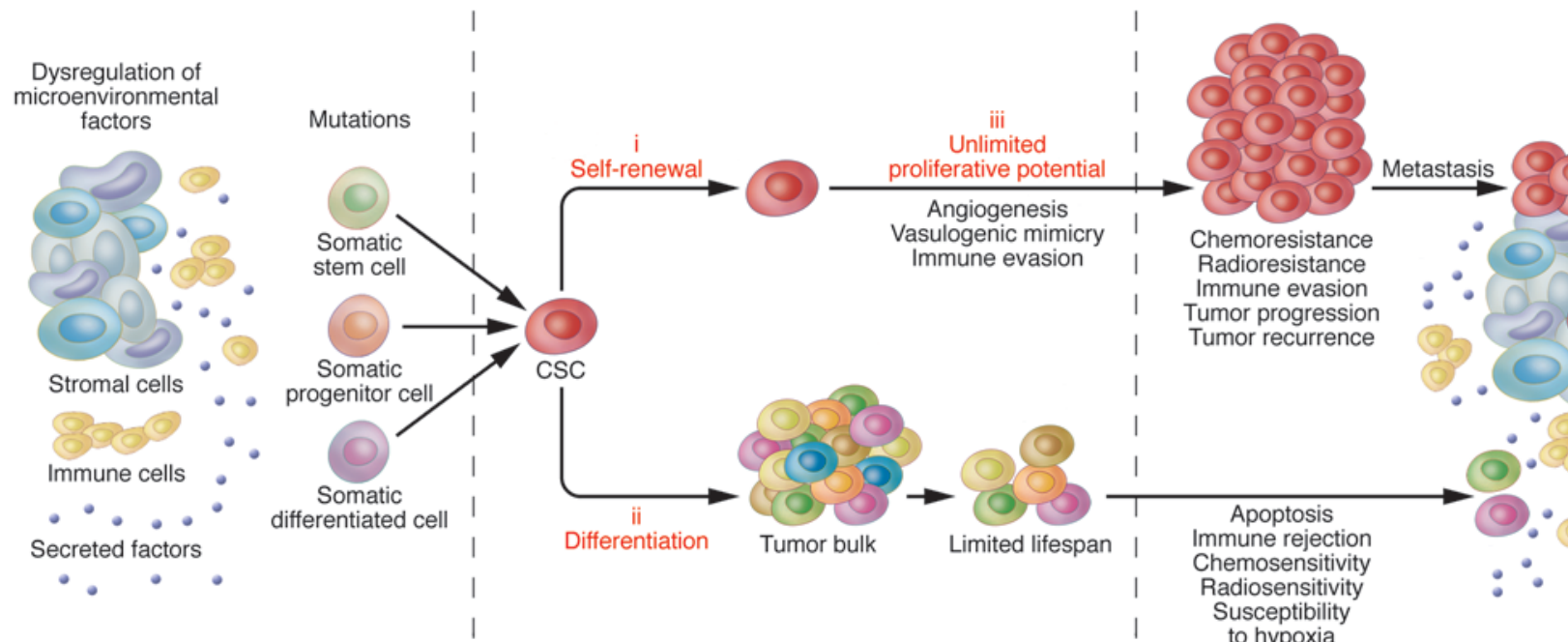

nlimited

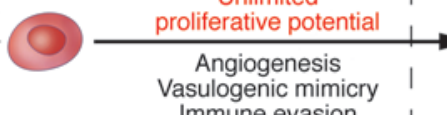
Immune evasion

I
I

Chemoresistance Radioresistance Immune evasion

Tumor progression Tumor recurrence

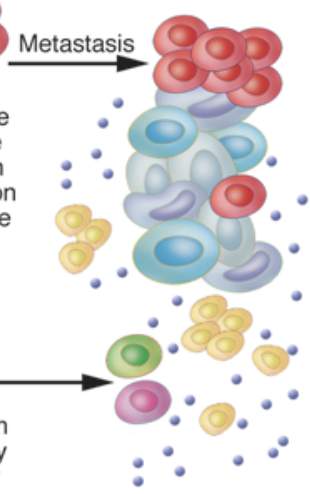

\section{Figure 1}

CSCs, carcinogenesis, tumorigenesis, and tumor resistance. (A) Tumors can arise from somatic cells through genetic mutations of cancer-critical genes. In addition, dysregulation of microenvironmental factors can contribute to the carcinogenic process. Such events might predominantly affect long-lived somatic stem cells, which can represent the cancer cell of origin, for example in mouse models of colorectal cancer (48). However, the CSC definition does not imply a specific relationship between CSCs and physiological stem cells. Findings in other disease models support progenitors or terminally differentiated somatic cell types as the source of malignant transformation. (B) CSCs are posited to be exclusively capable of driving tumorigenesis through 3 defining features: (i) their ability for long-term self-renewal, (ii) their capacity to differentiate into tumor bulk populations devoid of CSC characteristics, and (iii) their unlimited potential for proliferation and tumorigenic growth. Furthermore, CSCs in certain malignancies possess the capacity to drive tumor angiogenic responses and/or to engage in vasculogenic mimicry, potential means of promoting tumor growth. In addition, immunoevasive features of CSCs might contribute to tumorigenesis and ultimately to tumor progression. (C) CSCs can exhibit increased resistance to chemotherapeutic agents and/or ionizing radiation. CSCs might also possess a preferential capacity to withstand immune-mediated rejection. If CSCs indeed represent the pool of resistant cells in human cancer patients, they likely also drive neoplastic progression, tumor recurrence, and metastasis. Although this hypothesis requires further validation, clinical tumor progression has already been correlated with CSC frequency in human melanoma patients.

from a stem cell, a committed progenitor, or even a terminally differentiated cell $(12,19-22)$ (Figure 1$)$.

Tumorigenic populations fulfilling the definition of CSCs have been identified in a number of human cancers, including leukemias (23-28), bladder cancer (29), breast cancer (30), CNS cancers (31), colon carcinoma (32-34), head and neck cancer (35), ovarian cancer (36), pancreatic cancer $(37,38)$, malignant melanoma (13), liver cancer (39), and Ewing sarcoma (40). It is currently not known whether all cancers contain subpopulations of CSCs. The molecular phenotypes that have been used for CSC identification purposes in the aforementioned human malignancies are summarized in Table 1. It should be noted that in some instances, these findings have given rise to controversy with regard to the markers or marker combinations used and their relevance to clinical disease in the respective malignancies. For example, with regard to breast cancer, one study that examined 136 clinical breast cancer specimens found that the prevalence of CD $44^{+} \mathrm{CD} 24^{-}$tumor cells showed no correlation with clinical outcome and survival (41), potentially questioning the translational relevance of the finding of Al-Hajj et al. that this phenotype identifies cells with tumorigenic potential in human breast cancer samples (30). Nevertheless, the study did indicate that the prevalence of $\mathrm{CD} 44^{+} \mathrm{CD} 24^{-}$tumor cells might favor metastatic disease (41). A separate study that also questioned the translational relevance of the findings of Al-Hajj et al. described molecular and phenotypic analyses of CD24+ and CD $44^{+}$ breast cancer cells and found no correlation between CD24 and/or
CD44 expression in primary invasive breast carcinomas with any specific tumor characteristics (42). This study also suggested that genetic differences between $\mathrm{CD} 24^{+}$and $\mathrm{CD} 44^{+}$cells within a given tumor indicate independent clonal evolution as a cause of tumor heterogeneity. However, we believe that findings of clonal evolution do not necessarily contradict the existence of CSCs in a given malignancy $(15,43)$, including in breast cancer $(30)$. With regard to CD133 as a marker of brain cancer CSCs (31), Beier et al. found that CD133- glioma cells also contain subpopulations capable of initiating tumors, termed CD133- glioma CSCs (44). However, the self-renewal potential of CD133- CSCs could not be rigorously examined in this study in serial xenotransplantation experiments due to a lack of surface markers that would have allowed specific purification of CD133- CSCs from the larger pool of all CD133glioma cells. In addition, a separate study indicated the possibility of interconversion of $\mathrm{CD} 133^{-}$and $\mathrm{CD} 133^{+}$glioma cell phenotypes (45), but it did not demonstrate that CD133- glioma cells represent CSCs according to the consensus definition (12). With regard to colon cancer, one study (46) raised questions regarding CD133 $(33,34)$ as a universal marker of colon CSCs, based on findings that CD133 can be widely expressed by human primary colon cancer epithelial cells and that CD133 expression did not identify the entire population of epithelial and tumor-initiating cells in human metastatic colon cancer (46). In this study, both CD $133^{+}$ and $\mathrm{CD} 133^{-}$metastatic tumor subpopulations were capable of long-term tumorigenesis in a NOD/SCID serial xenotransplanta- 
Table 1

CSCs in human malignancies

\begin{tabular}{|c|c|c|}
\hline Malignancy & Molecular phenotype & Reference \\
\hline AML & $\mathrm{CD}_{4}{ }^{+} \mathrm{CD} 38^{-}$ & 23 \\
\hline Bladder & Lin-CD44+CK5+CK20- & 29 \\
\hline Breast & CD44+CD24-110 Lin-EPCAM+ & 30 \\
\hline CNS & CD133+ & 31 \\
\hline Colon & CD133+ & 33 \\
\hline Colon & CD133+ & 34 \\
\hline Colon & EPCAM ${ }^{\text {hiCD44+Lin-(CD166+) }}$ & 32 \\
\hline Ewing & $\mathrm{CD}_{133^{+}}$ & 40 \\
\hline Head and neck & CD44+Lin- & 35 \\
\hline Liver & $\mathrm{CD}^{+} 0^{+}$ & 39 \\
\hline Melanoma & ABCB5+ & 13 \\
\hline Ovarian & CD44+CD117+ & 36 \\
\hline Pancreatic & $\mathrm{CD}_{4}{ }^{+}{ }^{+}$24 ${ }^{+}$EPCAM $^{+}$ & 38 \\
\hline Pancreatic & CD133+ & 37 \\
\hline
\end{tabular}

CK5, cytokeratin 5.

tion model. These results indicate that CD133 may not uniformly identify colon CSCs in all tumor types and patients.

In addition to cell sorting-based CSC identification efforts, marker-specific genetic lineage tracking of subpopulations of cancer cells in competitive tumor development models has recently been used to further establish in vivo evidence for the existence of tumor hierarchies driven by molecularly defined CSCs (13). This approach has provided the additional advantage of detecting potential interactions between CSCs and bulk tumor cell populations that may occur in clinical cancers and was designed and used by our laboratory to identify CSCs in human malignant melanoma (13). Based on the demonstrated utility and feasibility of the genetic lineage tracking approach for CSC identification in human malignant melanoma and the success of similar cell fate tracking techniques as part of CSC identification strategies in human breast and brain cancer (47) as well as in models of colon carcinoma tumorigenesis $(48,49)$, we believe that in vivo genetic lineage tracking should be routinely included in the experimental repertoire to assay and validate CSC identity and function in human malignancies. Moreover, we believe it to be extremely important in light of the potential limitations of CSC assays that rely exclusively on sorted, untracked cancer cell subpopulations, as a recent study has shown that expression of 1 putative CSC marker changes as various malignant subpopulations cycle (50). In vivo genetic lineage tracking assays provide the additional advantage of facilitating potential niche interactions between CSCs and tumor bulk populations that may be operative in naturally occurring cancers but may typically evade detection when purified, isolated subpopulations of cells are studied (13).

The ability to prospectively identify CSCs has permitted researchers to begin characterizing specific molecular and cellular phenotypes and mechanisms preferentially associated with CSCs that may contribute to tumor initiation, growth, and progression, in addition to those associated with their defining features of unlimited self-renewal and proliferative capacities (Figure 1). As we discuss in this Review, among the recently uncovered CSC characteristics likely to influence tumor initiation, growth, and progression are functions associated with angiogenesis and vasculogenic mimicry as well as tumor immune evasion. These CSC interactions with the microenvironment and antitumor immune response of the tumor host might be susceptible to therapeutic intervention and therefore represent emerging foci of investigational interest and translational significance. Furthermore, we discuss direct CSC targeting approaches, for which therapeutic proof of principle has recently been established (13).

\section{CSC functions in tumorigenesis}

CSC-associated angiogenesis and vasculogenic mimicry. In 1971, Judah Folkman advanced the hypothesis of cancer angiogenesis, the notion that tumors are critically dependent upon tumor-related blood vessel growth and development (51). Initially controversial, this concept is now broadly accepted for its biologic and therapeutic significance. The precise pathways by which cancers stimulate angiogenesis (i.e., formation of new vessels from preexisting vessels; refs. 52, 53) in the peritumoral stroma, and the mechanisms responsible for the angiogenic response to tumor cells, have since been subjects of intense study, and a specific role for CSCs in angiogenesis has been identified recently (54). Furthermore, although it is thought that the tumor vasculature is mostly composed of nonmalignant endothelial cell populations originating from host angiogenesis $(52,53)$, there is evidence that tumor cells with high degrees of differentiation plasticity might contribute to the tumor vasculature via a process termed vasculogenic mimicry (55), which seems to mimic vasculogenesis (i.e., de novo vessel formation that occurs independently of angiogenesis through the de novo production of endothelial cells). In the case of human melanoma, CSCs have been found to be responsible for vasculogenic mimicry (13).

With regard to angiogenesis, CSCs can reciprocally modulate their microenvironment through either the secretion of paracrine factors or direct cell-cell contact. For example, in human brain cancer, CD $133^{+}$Nestin $^{+}$CSCs in medulloblastomas, ependymomas, oligodendrogliomas, and glioblastomas have been found to reside within a perivascular niche, where they interact closely with endothelial cells (56). Moreover, cografting endothelial cells in orthotopic human medulloblastoma tumor xenografts, induced - in a dose-dependent fashion - expansion of the self-renewing CSC fraction and accelerated cancer initiation and growth (56). In another study (54), a specific mechanistic relationship between CSCs and angiogenesis was established in glioma, in which glioma-initiating cells, enriched from clinical specimens using the cell surface marker CD133, were shown to specifically promote tumor angiogenesis, and thereby tumor xenograft growth, through the secretion of higher levels of VEGF compared with bulk tumor populations. Importantly, VEGF-specific neutralizing $\mathrm{mAb}$ treatment suppressed the growth of the $\mathrm{CD} 133^{+}$human glioma cell-derived xenografts (54), which indicates that this CSC function may represent a potential therapeutic target. In addition, Calabrese et al. have demonstrated that depletion of vascular endothelial cells, which were found to be capable of enhancing the in vivo self-renewal capacity of $\mathrm{CD} 133^{+}$brain CSCs through inhibition of either ERBB2 or VEGF signaling, reduced CSC abundance and substantially inhibited tumor xenograft growth (56). Further studies revealed that glioma CSCs responded differently to angiogenesis-promoting hypoxia, with distinct HIF response patterns, than did non-stem tumor cells and normal neural progenitors (57). Specifically, HIF2 $\alpha$ and multiple HIF-regulated genes were preferentially expressed in glioma CSCs. In addition, the integrity of the HIF response was functionally required for glioma CSC selfrenewal, proliferation, and survival in vitro and tumor initiation 
potential in vivo. Importantly, HIF2 $\alpha$ expression was also found to correlate with poor survival in human patients with a glioma (57), which indicates that HIF $2 \alpha$ might represent a promising target for antiglioblastoma therapies.

With regard to vasculogenic mimicry, Mary Hendrix and coworkers described in 1999 a phenomenon whereby human melanomas developed patterned networks of basement membrane that stained with periodic acid-Schiff and perfusable channels lined by tumor cells that expressed some, but not all, endothelial protein and genomic markers (58). They used the term vasculogenic mimicry to emphasize that the channels that formed were not lined by true endothelial cells, as is the case in vasculogenesis. It remains unknown whether vasculogenic mimicry represents a mechanism of tumor perfusion whereby aggressive and metabolically active tumors obtain the nutrients requisite for critical stages of growth and evolution and whether it is related to, or independent of, angiogenesis. However, it has been established that vasculogenic mimicry characterized by expression of tyrosine kinase with Ig-like and EGF-like domains 1 (TIE-1) and CD144 relates to melanoma aggressiveness (59) and that vasculogenic mimicry networks that also express laminin represent, in the case of human melanomas, a biomarker associated with increased clinical mortality (60). Indeed, vasculogenic mimicry has been proposed to provide a potential target for therapeutic intervention (59). In addition to detection in uveal, cutaneous, and mucous membrane melanomas, vasculogenic mimicry has also been identified in inflammatory and ductal breast carcinomas, ovarian carcinomas, prostate carcinomas, and soft tissue sarcomas, including synovial sarcoma, rhabdomyosarcoma, osteosarcoma, and pheochromocytoma (61).

Interestingly, addition of inhibitors of angiogenesis to melanoma cell cultures in vitro has been shown not to affect the tubular network formation typically associated with vasculogenic mimicry, whereas angiogenesis by HUVECs was abrogated under similar conditions (62). These findings suggest that vasculogenic mimicry might represent an important survival mechanism that could explain the failure of currently available inhibitors of angiogenesis to fully effect tumor eradication (63). Importantly, in the case of melanoma, tumor cell-driven vasculogenic mimicry has been linked to bone morphogenetic proteins (BMPs; ref. 64), secreted morphogen members of the TGF- $\beta$ superfamily that play critical roles in early embryonic vascular development (65). Indeed, Rothhammer et al. showed that impaired BMP activity resulted in reduced tumor growth in vivo in murine models of melanoma (64). In addition, others have shown that BMP4 signaling via bone morphogenetic protein receptor, type IA (BMPR1A) could regulate the size of the CSC population in human glioblastomas (66). However, the possibility that CSCs are selectively responsible for vasculogenic mimicry had not been considered until recently.

We recently found that human melanoma cells expressing $\mathrm{ABC}$, subfamily B (MDR/TAP), member 5 (ABCB5) represent a subpopulation of cells enriched for CSCs (13). Furthermore, our results demonstrated that in both clinical patient tumors and experimental tumors in serial xenotransplantation experiments, $\mathrm{ABCB} 5^{+} \mathrm{CSC}$ preferentially expressed the vasculogenic markers TIE- 1 and CD144 as well as BMPR1A (13). These findings suggested that the CSC compartment within a tumor could be responsible for vasculogenic mimicry and that this function of CSCs might represent one role by which CSCs can drive tumor formation, growth, and progression (67).

Further investigation of the relationship between CSCs and tumor angiogenesis and vasculogenic mimicry, and dissection of the cellular and molecular mechanisms involved, could provide opportunities for the development of novel CSC-targeted antiangiogenic therapies with advantages over currently available conventional antiangiogenic therapies. Indeed, despite great promise, conventional VEGF-targeted therapies have shown an unexpectedly limited survival benefit in the clinic (68), and this had led to additional studies aimed at further dissecting the effects of VEGF inhibition on primary tumor growth and tumor metastatic potential. In this regard, Ebos et al. found in a mouse model of breast cancer that, despite inhibitory effects of the VEGFR tyrosine kinase inhibitor sunitinib on established primary tumor growth, administration of sunitinib prior to or shortly after tumor cell inoculation resulted in accelerated tumor metastasis and decreased survival (69). Similar effects of VEGF inhibition have been reported in a model of pancreatic cancer, in which prolonged treatment of tumor-bearing mice with antiangiogenic drugs resulted in increased local tumor cell invasion and enhanced distant metastasis (70). Although the exact mechanisms underlying these findings remain to be elucidated, recent studies have identified important differences between inhibition of myeloid cell-derived VEGF compared with tumor-derived VEGF on tumor growth $(71,72)$. Specifically, targeting of myeloid cell-derived VEGF signaling leads to decreased phosphorylation of VEGFR2 and accelerated tumor progression as a result of tumor vasculature normalization (71), whereas targeting of both myeloid cell- and tumor-derived VEGF leads to collapse of tumor growth (72). Because CSCs can represent the major source of tumor-derived VEGF (54), specific targeting of CSCs or CSCderived VEGF production might represent a more promising form of antiangiogenic therapy compared with current modalities.

Immune evasion and modulation. In addition to tumor-promoting roles in angiogenesis and vasculogenic mimicry, CSCs might preferentially initiate and sustain neoplastic growth and disease progression through immunoevasive and immunomodulatory functions. This possibility is supported by findings that suggest negative correlations between the immune competence of the tumor host and rates of tumor formation. For example, markedly increased cancer risks have been described in immunocompromised human patients (73). Similarly, in experimental model systems, immunocompromised animals such as SCID and $\mathrm{Rag}^{-/-}$ mice, which each lack T and B cells, exhibit substantially increased rates of spontaneous malignancies (74). Furthermore, fewer tumor cells are required to initiate tumor growth in profoundly immunocompromised xenograft recipients $(14,23)$ than in less severely immunocompromised hosts $(13,75)$. Specifically, inocula of at least $2 \times 10^{5} \mathrm{CD} 34^{+} \mathrm{CD} 38^{-}$human acute myeloid leukemia (AML) cells were required to initiate leukemic disease in SCID mice (75), whereas only $5 \times 10^{3}$ cells were needed to cause leukemia in more severely immunocompromised NOD/SCID hosts (23). In addition, $\mathrm{CD} 34^{+} \mathrm{CD} 38^{-}$AML cells could be serially passaged to form secondary neoplasms in NOD/SCID mice (23), but not in the less severely immunocompromised SCID hosts (75), pointing to interdependence of CSC phenotype and function and recipient immune status in this malignancy. However, patient-dependent differences in AML CSC frequencies alone might account for the observed differences in these studies, because no direct comparison of AML xenografts from the same individuals to NOD/SCID and SCID recipients was performed in these studies $(23,75)$. In contrast, tumorigenicity clearly depends on host immunocompetence in human malignant melanoma $(13,14)$. CSC frequencies 
in human to NOD/SCID mouse xenotransplantation experiments have been estimated to be approximately 1 in $10^{6}$ tumor cells when tumor formation was assessed 8 weeks after xenotransplantation $(13,14)$. On the other hand, higher frequencies of cells capable of tumor initiation have been observed in side-by-side comparative studies in more severely immunocompromised xenograft recipients, NOD/SCID mice lacking the common cytokine receptor $\gamma$-chain ( $\gamma$ c; also known as IL-2R $\gamma$; ref. 14). These results demonstrate heterogeneity among cancer cells with regard to evasion of the host antitumor immune response and show that IL-2R $\gamma-$ dependent host immune effector responses eliminate some, but not all, melanoma cells capable of tumor initiation $(13,14)$, thereby indicating the existence of an immunoevasive subpopulation of CSCs (17). Moreover, these results indicate that CSC interactions with host immunity might contribute to the variability of experimentally determined CSC frequencies in human to mouse xenotransplantation models with varying degrees of host immunocompetence $(13-15,17,18)$, and that CSC immunomodulatory functions might lead to overestimation of CSC frequencies when clinically highly immunogenic cancers, such as human malignant melanoma $(67,76)$, are studied in profoundly immunocompromised experimental hosts, such as $I \mathrm{l}^{2 \mathrm{rg}^{-/}}$NOD/SCID mice (17, $18)$. The possibility that non-CSC populations, which do not normally initiate tumors, might cause experimental tumor growth in such models is suggested by clinical findings of detectable circulating tumor cell populations that frequently do not initiate tumors in human melanoma patients with relatively intact immunity (77). This is consistent with the view that it is possible - depending on how different the tumor environment is within patients compared with mouse models - that different cancer cells form tumors in mouse models than in human patients (14). Furthermore, it is possible that, despite observations of a high percentage of melanoma cells with the potential to proliferate extensively and form new tumors in particular models, an even greater, or a much smaller, fraction of melanoma cells actually contributes to disease progression in patients (14). Thus, while it is conceivable that model systems could be designed that allow most cancer cells to form an experimental tumor, it is likely that those models that account for, but do not mask, CSC-specific functions related to host microenvironmental and immune interactions might be more relevant to the study of clinically and translationally important CSC populations. Such models might consist, for example, of chimeric murine xenograft recipients: mice that are orthotopically xenografted with human cancer cells into syngeneic human tissue of cancer origin, already xenografted from the same patient, in the presence of an adoptively transferred hematopoietic system originating from the same donor that is capable of syngeneic antitumor immunity. This might be feasible, particularly in the case of human melanomas, for which chimeric orthotopic xenotransplantation models have previously been established (78).

The findings of higher rates of cancer development in immunocompromised patients and animal models imply that host immunosurveillance might serve to eliminate malignant cells at early stages of tumorigenesis, potentially explaining the relatively low frequency of tumor development in healthy individuals (79). The mechanisms by which CSCs might attenuate antitumor immune responses $(17,80,81)$ are currently unknown, but could include immunoregulatory mechanisms known to be operative in physiologic stem cells $(17,82-85)$, for example, contact-dependent mechanisms requiring engagement of the inhibitory molecule pro- grammed cell death-1 and secretion or induction of soluble immunomodulatory factors that are required for immunosuppression, such as TGF- $\beta 1$ and IL-10. Initial evidence for an immunoevasive phenotype of CSCs has been provided by recent findings in human malignant melanoma (17). This study showed that among A375 human melanoma cells, which, despite clonal origin, are heterogeneous for $\mathrm{ABCB} 5$ expression, the $\mathrm{ABCB} 5^{+} \mathrm{CSC}$ subpopulation selectively lacks the immunogenic melanoma-associated antigen recognized by $\mathrm{T}$ cells (MART-1; ref. 17). Thus, $\mathrm{ABCB5}^{+}$CSCs can possess a phenotype of relative immune privilege that suggests resistance to MART-1-directed immunotherapeutic treatment strategies. Accordingly, the possibility of CSC-driven tumor escape from immune-mediated rejection has important implications for current cancer immunotherapy and might represent a resistance mechanism susceptible to therapeutic intervention.

\section{Translational relevance of CSCs as targets for therapy}

Conventional anticancer approaches are directed predominantly at bulk tumor populations. Such strategies often have limited efficacy because of intrinsic or acquired drug resistance and/or resistance to ionizing radiation (86). Mechanisms of therapeutic resistance include increased recognition and repair of DNA damaged by the drug or ionizing radiation, altered cell cycle checkpoint control, impaired functioning of apoptotic pathways, and reduced drug accumulation as a result of increased expression of $\mathrm{ABC}$ transporters that efflux drug $(86,87)$. Evidence has emerged that CSCs represent a subpopulation of cells within cancers that is characterized by increased resistance to chemo- and radiotherapy, indicating that conventional anticancer approaches might frequently fail to eradicate the cell subset that initiates and perpetuates tumorigenesis (Figure 1). For example, CSC chemoresistance has been reported in human leukemias $(27,80,88-92)$, in malignant melanoma $(13,93)$, and in brain $(94)$, breast $(95,96)$, pancreatic (37), and colorectal (97) cancers. Furthermore, CSC radioresistance has been identified in brain $(98)$ and breast $(99,100)$ cancers. If CSCs are indeed the major culprits of tumor development and responsible for therapeutic resistance and malignant progression in human patients, treatment approaches that target CSCs could potentially increase the efficacy of currently available treatment regimens and reduce the risk of tumor relapse and metastasis.

Proof of principle for the potential therapeutic efficacy of CSC targeting requires evaluation of the in vivo tumor-inhibitory effects of therapies directed at CSCs molecularly defined by a prospective marker not expressed by nontumorigenic bulk cancer populations (13). Such approaches are warranted because antitumor effects of therapies directed at both CSCs and bulk cancer populations cannot be ascribed with certainty to an effect on either cell population alone. Accordingly, provision of this proof of principle is currently not experimentally feasible in circumstances in which candidate CSC populations have been identified through marker combinations that include positivity for molecules also expressed by most of the tumor cells and negativity for molecules expressed by bulk cancer populations, for example, positivity for CD34 and negativity for CD38 of CSCs in AML (Table 1 and ref. 23). In contrast, it is feasible in those malignancies in which CSCs have been reported to be enriched among cell populations identified by a single prospective molecular marker not shared by the bulk cancer populations, for example, the CSCs in brain cancer (31), colon cancer (33, 34), pancreatic cancer (37), and Ewing sarcoma (40), all of which have been identified by expression of CD133; the CSCs in liver can- 


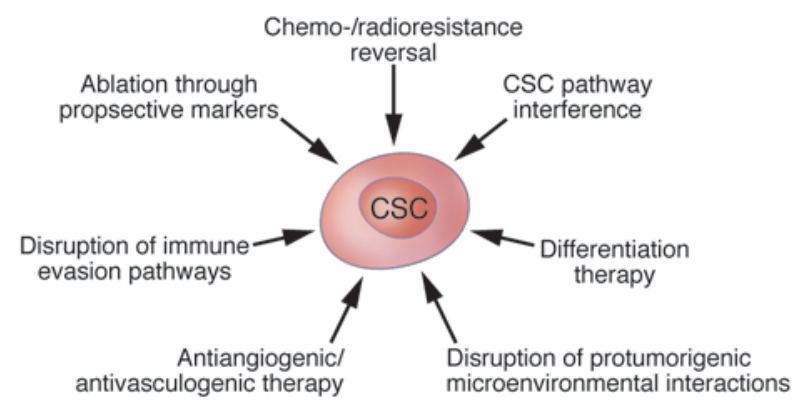

Figure 2

The therapeutic promise of CSC-directed targeting strategies. A number of therapeutic strategies directed at CSCs are beginning to be experimentally validated. These approaches could potentially enhance responsiveness to current anticancer treatment regimens and might reduce the risk of relapse and dissemination. The approaches include ablation using antitumor agents that target prospective markers of CSCs (e.g., monoclonal antibodies and activated immune cells); reversal of chemo- or radioresistance mechanisms operative in CSC; CSC pathway interference; differentiation therapy; disruption of protumorigenic CSC-microenvironment interactions; antiangiogenic or antivasculogenic therapy; and disruption of immunoevasion pathways.

cer identified by expression of CD90 (39); and the CSCs in human malignant melanoma, identified by expression of ABCB5 (Table 1 and ref. 13). Indeed, initial proof of principle for the potential therapeutic utility of the CSC concept has recently been provided in human melanoma by the demonstration that selective killing of the CSC subpopulation identified by ABCB5 expression is sufficient to inhibit experimental tumor growth (13). These findings support the therapeutic promise of CSC-targeted approaches in human malignant melanoma. Furthermore, they provide a rationale for the development of additional therapeutic strategies directed at targeting functionally relevant molecular pathways in CSCs in other cancers, regardless of whether such pathways might be exclusively associated with CSC subsets or whether they are operative in both CSCs and bulk cancer populations. Indeed, those therapeutics that target both CSCs and cancer bulk populations might prove most effective for tumor eradication.

Several CSC-targeted approaches harbor promise for increasing cancer therapeutic efficacy (Figure 2). In addition to the aforementioned potential indirect strategies related to the angiogenic/vasculogenic functions and the immunoevasive properties of CSCs, there are direct strategies, such as CSC ablation using agents that target their molecular markers, reversal of resistance mechanisms operative in CSCs, and differentiation therapy. It is important to recognize in this regard that the CSC phenotype can display both intra- and interindividual variability in particular malignancies and that CSC-directed therapies might therefore have limitations with regard to targeting every CSC in all patients.

CSC ablation using agents that target their molecular markers and signaling pathways. Abundance of the CSC subset identified by expression of the chemoresistance mediator ABCB5 correlates positively with neoplastic progression in human melanoma patients (13). Consistent with this, the $A B C B 5$ gene is also preferentially expressed by melanomas with high in vivo tumorigenic capacity in human to murine xenotransplantation models $(101,102)$ and by melanomas of metastatic tumor origin, but not those that are primary tumors (103). Thus, ABCB5 provides a direct and unique link among CSCs, resistance to anticancer therapeutics, and neoplastic progression in human malignant melanoma. We therefore examined whether targeted ablation of a prospectively identified CSC compartment inhibits tumor growth (13). Interestingly, administration of an anti-ABCB5 mAb to nude mouse recipients of human melanoma xenografts substantially inhibited tumor formation and growth when started prior to xenotransplantation. Furthermore, the antiABCB5 mAb limited the growth of established tumors compared with control xenograft recipients, through CSC-specific Ab-dependent cell-mediated cytotoxicity (13). These findings have provided initial proof of principle that targeted ablation of CSCs using an agent directed at a CSC-defining molecular marker is sufficient to substantially inhibit tumorigenesis and tumor growth (13). Also in human malignant melanoma, shRNA-mediated knockdown of CD133 - which is expressed on subsets of human melanoma cells $(93,104,105)$, including a subset of $\mathrm{ABCB5}^{+} \mathrm{CSCs}(93)$ - resulted in reduced melanoma clonogenicity and motility in vitro and in inhibition of melanoma metastatic potential in experimental model systems in vivo (106).

Direct CSC killing by targeting a CSC-expressed molecular marker has also been reported in experimental models of hepatocellular carcinoma (39). In this malignancy, Yang et al. identified a population of CSCs expressing CD90 that showed an increased capacity to initiate experimental tumors compared with CD90- cancer cells in a human to nude mouse xenotransplantation model (39). Among this CSC population, a subpopulation of $\mathrm{CD} 90^{+} \mathrm{CD} 44^{+}$cells demonstrated a phenotype more aggressive than that of $\mathrm{CD} 90^{+} \mathrm{CD} 44^{-}$cells, characterized by formation of metastatic lesions in the lungs of immunodeficient mice. Systemic administration of a human CD44-specific mAb at the time of subcutaneous $\mathrm{CD} 90^{+} \mathrm{CD} 44^{+}$carcinoma cell inoculation markedly inhibited tumor initiation and growth compared with controls. Quantification of $\mathrm{CD} 90^{+} \mathrm{CD} 44^{+}$tumor cell apoptosis after in vitro treatment with the human CD44-specific mAb suggested mAbinduced CSC apoptosis as a possible mechanism underlying the observed inhibition of tumor xenograft formation (39).

In human gliomas, shRNA-mediated knockdown of the neural cell adhesion molecule L1 cell adhesion molecule (L1CAM), which is preferentially expressed on $\mathrm{CD} 133^{+}$glioma CSCs, substantially decreased the ability of these CSCs to form neurospheres (a nonadherent in vitro growth pattern associated with the CSC phenotype) and induced apoptosis of $\mathrm{CD}_{133^{+}}$, but not CD133-, glioma cells in vitro (107). In vivo, L1CAM knockdown in $\mathrm{CD}_{133^{+}}$glioma cells prior to xenotransplantation into immunodeficient mice markedly inhibited in vivo tumorigenesis and prolonged survival of the tumor xenograft recipients. Furthermore, intracranial administration of lentiviral preparations expressing L1CAM shRNA to immunocompromised mice 5 days after $\mathrm{CD} 133^{+}$glioma cell xenotransplantation also substantially suppressed tumor growth and prolonged survival of the tumor-bearing mice (107). In addition, Vlashi et al. have reported that, among human glioma cells and human breast cancer cells, malignant subpopulations that exhibit CSC characteristics and preferential resistance to ionizing radiation can be identified by reduced $26 \mathrm{~S}$ proteasome activity compared with bulk tumor populations (47). Targeted killing of these cells via a proteasome-dependent thymidine kinase suicide gene resulted in experimental tumor regression (47), which suggests that differences in proteasome activity represent potential targets for CSC-directed cancer therapy. 
In human bladder cancer, CSCs were recently identified as $\mathrm{Lin}^{-} \mathrm{CD} 44^{+} \mathrm{CK}^{+} \mathrm{CK} 20^{-}$(29). This study also revealed that CD47, a protein that provides an inhibitory signal for macrophage phagocytosis, is more highly expressed by bladder CSCs than the rest of the tumor cells. Blockade of CD47 using a mAb resulted in engulfment of the CSCs by macrophages and subsequent bladder cancer cell killing in vitro (29), which points to a potential therapeutic value of this CSC-targeted approach for invasive bladder cancer if similar effects of $\mathrm{CD} 47$-specific $\mathrm{mAb}$ are found in in vivo preclinical studies (29).

In human leukemias, inhibition of NF-кB induced apoptosis of $\mathrm{CD} 34^{+} \mathrm{CD} 38^{-} \mathrm{CSC}$ in vitro and inhibited tumor growth in experimental animal models in vivo, while sparing the physiologic HSC compartment (108). Furthermore, also in human leukemia, expression of the $\alpha$ subunit of the IL-3 receptor (CD123) was detected at higher levels on both AML CD $34^{+} \mathrm{CD} 38^{-}$stem cells and bulk AML populations compared with normal hematopoietic cells (109). Treatment of human AML cells with a CD123-specific neutralizing $\mathrm{mAb}$ resulted in impaired cancer cell engraftment and proliferation in murine NOD/SCID xenograft hosts and improved longterm survival through targeting both leukemic stem cells and bulk populations (109). Leukemic CSCs have also been targeted through modulation of the PI3K/phosphatase and tensin homolog/mammalian target of rapamycin (PI3K/PTEN/mTOR) pathway in a mouse model of leukemia (110). Conditional deletion of the Pten tumor suppressor gene in adult hematopoietic cells induced transplantable leukemias in which leukemia-initiating cells could be depleted by rapamycin through inhibition of mTOR (110).

In aggregate, these results demonstrate the feasibility and therapeutic promise of CSC ablation through targeting molecular markers specifically expressed by CSCs or expressed by both CSCs and bulk cancer cell populations. MAb- and shRNA-based strategies may prove to be especially useful in this regard because of their high degrees of target specificity.

Reversal of resistance mechanisms operative in CSCs. Experimental evidence has been generated in several human malignancies that inhibition of the mechanisms responsible for CSC resistance to chemo- and radiotherapy represents a promising CSC-directed therapeutic strategy. Reversing chemoresistance in CSC populations can be achieved through specific blockade of multidrug resistance $\mathrm{ABC}$ transporters, as shown in human melanoma (93). The CSC marker ABCB5 mediates melanoma resistance to the chemotherapeutic agent doxorubicin $(93,111)$, and this effect is reversible by both $\mathrm{mAb}$-mediated inhibition of ABCB5-dependent drug efflux (93) and siRNA-mediated $A B C B 5$ gene silencing (111). In addition, $A B C B 5$ gene silencing increases substantially the sensitivity of human melanoma cells to the anticancer chemotherapeutics 5-fluorouracil (5-FU) and camptothecin (112). A potential broader role for $\mathrm{ABCB} 5$ in anticancer chemotherapeutic resistance is suggested by the observation that across a panel of human cancer cell lines used by the National Cancer Institute for drug screening, $A B C B 5$ gene expression levels correlate with chemoresistance to 45 of 119 anticancer agents (93), and the finding that human chronic myeloid leukemia cells resistant to the anticancer chemotherapeutic vincristine exhibit $A B C B 5$ gene amplification and enhanced expression $(113,114)$. These results warrant further in vivo examination to determine whether ABCB5-targeted sensitization to clinically relevant therapeutic agents represents a feasible and effective strategy to eradicate chemoresistant CSCs. Recent findings in colon cancer further support the potential therapeutic utility of CSC chemosensitizing agents (115). In this study, the researchers demonstrated that pretreatment of $\mathrm{CD}_{133^{+}}$colon CSCs with an IL-4-specific neutralizing Ab enhanced apoptosis mediated by 5 -FU and oxiplatin in vitro and in xenotransplanted immunodeficient mice (115).

CSC-targeted therapeutic approaches might also include strategies directed at reversal of radioresistance. In recent years, several studies have reported that CSCs have increased radioresistance compared with bulk cancer populations and proposed that reversal of CSC radioresistance might be critical for curing solid cancers for which radiation remains the first-line treatment (116). Bao et al. reported that, in human gliomas, CD $133^{+}$glioma CSCs contributed to tumor radioresistance through preferential activation of the DNA damage checkpoint response and through increased DNA repair capacity (98). In this study, inhibiting the Chk 1 and Chk 2 checkpoint kinases reversed the radioresistance of $\mathrm{CD}_{133^{+}}$glioma CSCs (98). Additional mechanisms of increased radioresistance in gliomas have been described by Chang et al. (117), who found that CD $133^{+}$glioma CSCs expressed higher levels of sirtuin 1 (SIRT1) compared with CD133- bulk cancer populations and that specific SIRT1 inhibition resulted in markedly enhanced radiosensitivity of $\mathrm{CD} 133^{+}$glioma CSCs. These results suggested that SIRT1 is a potential target for reversing glioma CSC resistance to radiotherapy (117). In breast cancer, increased radioresistance of $\mathrm{CD} 24^{-/ \mathrm{lo}} \mathrm{CD} 44^{+} \mathrm{CSC}$ has been attributed to decreased production of ROS in response to radiation as a result of high expression levels of free-radical scavengers $(99,100)$ and activation of the Notch signaling cascade (100). Consistent with the former, pharmacologic depletion of ROS scavengers resulted in radiosensitization (99). In aggregate, these studies indicate that targeting mechanisms of radioresistance operative in CSCs represents a promising approach to ultimately facilitate tumor eradication and prevent cancer recurrence.

Differentiation therapy. The possibility that differentiation of more primitive cells within a malignancy may lead to tumor degeneration and increased susceptibility to conventional cytotoxic anticancer therapies has been recognized for some time (118). Therefore, differentiation therapy holds promise as an approach to target CSCs. Potential strategies that induce quiescent CSCs to differentiate into more mature tumor cells include activation of distinct signaling pathways, such as morphogen-driven signaling cascades (66); alteration of gene expression profiles using microRNAs (miRNAs; ref. 96); and epigenetic differentiation therapy $(119,120)$. Piccirillo et al. have harnessed BMP signaling to differentiate CSCs in experimental models of human glioblastoma (66). Administration of BMP4 to human cancer-bearing mice induced glioblastoma differentiation and markedly attenuated CD $133^{+}$ CSC frequency (66). In addition, implantation of BMP4-treated glioblastoma xenografts to murine recipients resulted in smaller tumor lesions compared with untreated controls and substantially prolonged host survival (66). Preferential expression of BMPR1A on $\mathrm{ABCB}^{+} \mathrm{CSC}$ in human melanomas (13) suggests that similar BMP4-dependent differentiation strategies might also hold therapeutic promise in this malignancy. In medulloblastoma, modulation of CSC signaling pathways has also been shown to induce CSC differentiation (121). Specifically, administration of Notch pathway inhibitors resulted in depletion of medulloblastoma stem-like cells. Small noncoding miRNAs have also been shown to be capable of regulating CSC differentiation and function. For example, in breast cancer, enforced expression of the let-7 miRNA induced 
differentiation of $\mathrm{CD} 44^{+} \mathrm{CD} 24^{-/ 10} \mathrm{CSCs}$ and inhibited their ability to form tumors in mice (96). Most recently, also in human breast cancer, Gupta et al. used a high-throughput screening approach to determine the anticancer activity of approximately 16,000 compounds and identified a potassium ionophore, salinomycin, that selectively targets $\mathrm{CD} 24^{\mathrm{lo}} \mathrm{CD} 44^{\mathrm{hi}} \mathrm{CSCs}$ (122). Treatment of mice with this agent induced epithelial differentiation of tumor cells and resulted in inhibition of tumor growth, pointing to a promising role for this approach in breast cancer therapy.

In human AML, Jin et al. (123) reported a therapeutic approach using an activating $\mathrm{mAb}$ directed to the adhesion molecule CD44. In vivo administration of this $\mathrm{Ab}$ to NOD/SCID mice transplanted with human AML markedly reduced leukemic repopulation. Absence of leukemia in serially transplanted mice demonstrated that AML CSCs were directly targeted. Mechanistically, CD44-specific $\mathrm{Ab}$ treatment was shown to induce differentiation to more mature cancer cell progeny that were unable to establish robust leukemia upon xenotransplantation (123). Interference with cancer cell transport to stem cell-supportive microenvironmental niches was found to represent an additional mechanism underlying CD44-specific Ab-mediated AML CSC eradication, indicating a role for the microenvironment in regulating CSC function (123).

Epigenetic therapy could potentially also be used to induce CSC differentiation, thereby rendering these aggressive cells more susceptible to conventional cytotoxic treatment. Indeed, inhibitors of DNA methyltransferases and/or histone deacetylases have been shown to induce CSC differentiation that bypasses cancer-associated genetic abnormalities (119). In recent clinical findings in patients with locally advanced breast cancer, a therapeutic regimen consisting of the demethylating agent hydralazine and the histone deacetylase inhibitor magnesium valproate followed by doxorubicin and cyclophosphamide therapy resulted in a trend toward improved clinical outcome compared with doxorubicin and cyclophosphamide therapy alone (124). The doxorubicin chemoresistance mediator ABCB5 $(93,111)$ is expressed in human breast cancer and was substantially downregulated after differentiation therapy in these studies (124), raising the possibility that loss of more primitive and doxorubicin-resistant ABCB5-expressing breast cancer cells might have contributed to the observed results. In aggregate, the results regarding differentiation therapy suggest that these CSC-targeted approaches hold promise for improving currently available forms of cancer therapy.

\section{Conclusion}

CSC-specific phenotypes and mechanisms that relate to functions in tumorigenicity, cancer progression, and therapeutic resistance have been identified. These results indicate that CSCs may contribute to the failure of existing therapies to consistently eradicate malignant tumors. Therefore, CSCs represent novel and translationally relevant targets for clinical cancer therapy. Importantly, proof-of-principle experiments have strengthened the rationale for developing CSC-targeted therapeutic modalities that might complement more conventional cancer therapies. Indeed, CSCtargeted approaches have shown promise in preclinical models. These approaches include direct strategies, such as ablation by targeting molecular markers of CSCs or CSC-specific pathways, reversal of resistance mechanisms, and differentiation therapy, and indirect strategies, such as antiangiogenic therapy, immunotherapeutic approaches, and disruption of protumorigenic interactions between CSCs and their microenvironment. In some instances, mAb-based strategies that target molecules expressed by CSCs, for example, epithelial cell adhesion molecule (EPCAM) on breast and colon CSCs (Table 1 and refs. 30, 32), are already being translated to the clinic (125). These developments underline the therapeutic promise of the CSC concept. Ultimately, patient cures will require eradication of all cells within a cancer; therefore, combination therapies that target both CSCs and bulk cancer populations are likely to emerge as particularly effective clinical strategies, especially in those malignancies currently refractory to conventional anticancer agents directed predominantly at the bulk tumor cell populations.

\section{Acknowledgments}

This work was supported by National Cancer Institute, NIH, grants 1RO1CA113796, 1R01CA138231, and 2P50CA093683-06A20006 (to M.H. Frank) and by National Institute of Neurological Disorders and Stroke, NIH, grant 1KO8NS051349 (to N.Y. Frank). T. Schatton is the recipient of a Postdoctoral Fellowship Award from the American Heart Association Founders Affiliate.

Address correspondence to: Markus H. Frank, Transplantation Research Center, Children's Hospital Boston and Brigham and Women's Hospital, Harvard Medical School, 300 Longwood Avenue, Boston, Massachusetts 02115, USA. Phone: (617) 919-2993, Fax: (617) 730-0129, E-mail: markus.frank@childrens.harvard.edu.
1. Reya T, Morrison SJ, Clarke MF, Weissman IL. Stem cells, cancer, and cancer stem cells. Nature. 2001;414(6859):105-111.

2. Morrison SJ, Kimble J. Asymmetric and symmetric stem-cell divisions in development and cancer. Nature. 2006;441(7097):1068-1074.

3. Scadden DT. The stem-cell niche as an entity of action. Nature. 2006;441(7097):1075-1079.

4. Fidler IJ, Hart IR. Biological diversity in metastatic neoplasms: origins and implications. Science. 1982;217(4564):998-1003.

5. Hanahan D, Weinberg RA. The hallmarks of cancer. Cell. 2000;100(1):57-70.

6. Griffin JD, Lowenberg B. Clonogenic cells in acute myeloblastic leukemia. Blood. 1986;68(6):1185-1195.

7. Sabbath KD, Ball ED, Larcom P, Davis RB, Griffin JD. Heterogeneity of clonogenic cells in acute myeloblastic leukemia. J Clin Invest. 1985;75(2):746-753.

8. Bruce WR, Van Der Gaag H. A quantitative assay for the number of murine lymphoma cells capable of proliferation in vivo. Nature. 1963;199:79-80.

9. Hamburger AW, Salmon SE. Primary bioas- say of human tumor stem cells. Science. 1977; 197(4302):461-463.

10. Park CH, Bergsagel DE, McCulloch EA. Mouse myeloma tumor stem cells: a primary cell culture assay. J Natl Cancer Inst. 1971;46(2):411-422.

11. Schatton T, Frank NY, Frank MH. Solid tumor stem cells - implications for cancer therapy. In: Rajasekhar VK, Vemuri MC, eds. Regulatory Networks in Stem Cells. New York, NY: Humana Press; 2009:527-544.

12. Clarke MF, et al. Cancer stem cells-perspectives on current status and future directions: AACR Workshop on cancer stem cells. Cancer Res. 2006; 66(19):9339-9344

13. Schatton $\mathrm{T}$, et al. Identification of cells initiating human melanomas. Nature. 2008;451(7176):345-349.

14. Quintana E, Shackleton M, Sabel MS, Fullen DR, Johnson TM, Morrison SJ. Efficient tumour formation by single human melanoma cells. Nature. 2008;456(7222):593-598.

15. Gupta PB, Chaffer CL, Weinberg RA. Cancer stem cells: mirage or reality? Nat Med. 2009;
15(9):1010-1012.

16. Orimo A, et al. Stromal fibroblasts present in invasive human breast carcinomas promote tumor growth and angiogenesis through elevated SDF-1/ CXCL12 secretion. Cell. 2005;121(3):335-348.

17. Schatton T, Frank MH. Antitumor immunity and cancer stem cells. Ann N Y Acad Sci. 2009; 1176:154-169.

18. Schatton T, Frank NY, Frank MH. Identification and targeting of cancer stem cells. Bioessays. 2009; 31(10):1038-1049.

19. Jamieson $\mathrm{CH}$, et al. Granulocyte-macrophage progenitors as candidate leukemic stem cells in blastcrisis CML. N Engl J Med. 2004;351(7):657-667.

20. Huntly BJ, et al. MOZ-TIF2, but not BCR-ABL, confers properties of leukemic stem cells to committed murine hematopoietic progenitors. Cancer Cell. 2004;6(6):587-596.

21. Krivtsov AV, et al. Transformation from committed progenitor to leukaemia stem cell initiated by MLL-AF9. Nature. 2006;442(7104):818-822.

22. Sun B, Chen M, Hawks CL, Pereira-Smith OM, 
Hornsby PJ. The minimal set of genetic alterations required for conversion of primary human fibroblasts to cancer cells in the subrenal capsule assay. Neoplasia. 2005;7(6):585-593.

23. Bonnet D, Dick JE. Human acute myeloid leukemia is organized as a hierarchy that originates from a primitive hematopoietic cell. Nat Med. 1997;3(7):730-737.

24. Castor A, et al. Distinct patterns of hematopoietic stem cell involvement in acute lymphoblastic leukemia. Nat Med. 2005;11(6):630-637.

25. Cox CV, Evely RS, Oakhill A, Pamphilon DH, Goulden NJ, Blair A. Characterization of acute lymphoblastic leukemia progenitor cells. Blood. 2004;104(9):2919-2925.

26. Cox CV, Martin HM, Kearns PR, Virgo P, Evely RS, Blair A. Characterization of a progenitor cell population in childhood T-cell acute lymphoblastic leukemia. Blood. 2007;109(2):674-682.

27. Ishikawa $\mathrm{F}$, et al. Chemotherapy-resistant human AML stem cells home to and engraft within the bone-marrow endosteal region. Nat Biotechnol. 2007; 25(11):1315-1321.

28. Matsui W, et al. Characterization of clonogenic multiple myeloma cells. Blood. 2004;103(6):2332-2336.

29. Chan KS, et al. Identification, molecular characterization, clinical prognosis, and therapeutic targeting of human bladder tumor-initiating cells. Proc Natl Acad Sci U S A. 2009;106(33):14016-14021.

30. Al-Hajj M, Wicha MS, Benito-Hernandez A, Morrison SJ, Clarke MF. Prospective identification of tumorigenic breast cancer cells. Proc Natl Acad Sci US A. 2003;100(7):3983-3988.

31. Singh SK, et al. Identification of human brain tumour initiating cells. Nature. 2004;432(7015):396-401.

32. Dalerba P, et al. Phenotypic characterization of human colorectal cancer stem cells. Proc Natl Acad Sci U S A. 2007;104(24):10158-10163.

33. O'Brien CA, Pollett A, Gallinger S, Dick JE. A human colon cancer cell capable of initiating tumour growth in immunodeficient mice. Nature. 2007;445(7123):106-110.

34. Ricci-Vitiani L, et al. Identification and expansion of human colon-cancer-initiating cells. Nature. 2007; 445(7123):111-115.

35. Prince ME, et al. Identification of a subpopulation of cells with cancer stem cell properties in head and neck squamous cell carcinoma. Proc Natl Acad Sci US A. 2007;104(3):973-978.

36. Zhang $S$, et al. Identification and characterization of ovarian cancer-initiating cells from primary human tumors. Cancer Res. 2008;68(11):4311-4320.

37. Hermann PC, et al. Distinct populations of cancer stem cells determine tumor growth and metastatic activity in human pancreatic cancer. Cell Stem Cell. 2007;1(3):313-323.

38. Li C, et al. Identification of pancreatic cancer stem cells. Cancer Res. 2007;67(3):1030-1037.

39. Yang ZF, et al. Significance of CD90(+) cancer stem cells in human liver cancer. Cancer Cell. 2008; 13(2):153-166.

40. Suva ML, et al. Identification of cancer stem cells in Ewing's sarcoma. Cancer Res. 2009;69(5):1776-1781.

41. Abraham BK, Fritz P, McClellan M, Hauptvogel $\mathrm{P}$, Athelogou M, Brauch H. Prevalence of CD44+/ CD24-/low cells in breast cancer may not be associated with clinical outcome but may favor distant metastasis. Clin Cancer Res. 2005;11(3):1154-1159.

42. Shipitsin M, et al. Molecular definition of breast tumor heterogeneity. Cancer Cell. 2007;11(3):259-273.

43. Shackleton M, Quintana E, Fearon ER, Morrison SJ. Heterogeneity in cancer: cancer stem cells versus clonal evolution. Cell. 2009;138(5):822-829.

44. Beier D, et al. CD133(+) and CD133(-) glioblastoma-derived cancer stem cells show differential growth characteristics and molecular profiles. Cancer Res. 2007;67(9):4010-4015.

45. Wang J, et al. CD133 negative glioma cells form tumors in nude rats and give rise to CD133 positive cells. Int J Cancer. 2008;122(4):761-768.

46. Shmelkov SV, et al. CD133 expression is not restricted to stem cells, and both $\mathrm{CD} 133^{+}$and CD133 metastatic colon cancer cells initiate tumors. J Clin Invest. 2008;118(6):2111-2120.

47. Vlashi E, et al. In vivo imaging, tracking, and targeting of cancer stem cells. J Natl Cancer Inst. 2009; 101(5):350-359.

48. Barker N, et al. Crypt stem cells as the cells-of-origin of intestinal cancer. Nature. 2009;457(7229):608-611.

49. Zhu L, et al. Prominin 1 marks intestinal stem cells that are susceptible to neoplastic transformation. Nature. 2009;457(7229):603-607.

50. Jaksch M, Munera J, Bajpai R, Terskikh A, Oshima RG. Cell cycle-dependent variation of a CD133 epitope in human embryonic stem cell, colon cancer, and melanoma cell lines. Cancer Res. 2008;68(19):7882-7886.

51. Folkman J. Tumor angiogenesis: therapeutic implications. N Engl J Med. 1971;285(21):1182-1186.

52. Folkman J. Seminars in Medicine of the Beth Israel Hospital, Boston. Clinical applications of research on angiogenesis. NEnglJMed. 1995;333(26):1757-1763.

53. Risau W. Mechanisms of angiogenesis. Nature. 1997;386(6626):671-674.

54 . Bao S, et al. Stem cell-like glioma cells promote tumor angiogenesis through vascular endothelial growth factor. Cancer Res. 2006;66(16):7843-7848.

55. Hendrix MJ, Seftor EA, Hess AR, Seftor RE. Vasculogenic mimicry and tumour-cell plasticity: lessons from melanoma. Nat Rev Cancer. 2003;3(6):411-421.

56. Calabrese C, et al. A perivascular niche for brain tumor stem cells. Cancer Cell. 2007;11(1):69-82.

57. Li Z, et al. Hypoxia-inducible factors regulate tumorigenic capacity of glioma stem cells. Cancer Cell. 2009;15(6):501-513.

58. Maniotis AJ, et al. Vascular channel formation by human melanoma cells in vivo and in vitro: vasculogenic mimicry. Am J Pathol. 1999;155(3):739-752.

59. Folberg R, Hendrix MJ, Maniotis AJ. Vasculogenic mimicry and tumor angiogenesis. Am J Pathol. 2000;156(2):361-381.

60. Lin AY, et al. Distinguishing fibrovascular septa from vasculogenic mimicry patterns. Arch Pathol Lab Med. 2005;129(7):884-892.

61. Folberg R, Maniotis AJ. Vasculogenic mimicry. APMIS. 2004;112(7-8):508-525.

62. van der Schaft DW, et al. Effects of angiogenesis inhibitors on vascular network formation by human endothelial and melanoma cells. J Natl Cancer Inst. 2004;96(19):1473-1477.

63. Folkman J. Angiogenesis: an organizing principle for drug discovery? Nat Rev Drug Discov. 2007;6(4):273-286.

64. Rothhammer T, Bataille F, Spruss T, Eissner G, Bosserhoff AK. Functional implication of BMP4 expression on angiogenesis in malignant melanoma. Oncogene. 2007;26(28):4158-4170.

65. Liu W, et al. Bmp4 signaling is required for outflowtract septation and branchial-arch artery remodeling. Proc Natl Acad Sci U S A. 2004;101(13):4489-4494.

66. Piccirillo SG, et al. Bone morphogenetic proteins inhibit the tumorigenic potential of human brain tumour-initiating cells. Nature. 2006; 444(7120):761-765.

67. Schatton T, Frank MH. Cancer stem cells and human malignant melanoma. Pigment Cell Melanoma Res. 2008;21(1):39-55.

68. Kerbel RS. Tumor angiogenesis. $N$ Engl J Med. 2008;358(19):2039-2049.

69. Ebos JM, Lee CR, Cruz-Munoz W, Bjarnason GA, Christensen JG, Kerbel RS. Accelerated metastasis after short-term treatment with a potent inhibitor of tumor angiogenesis. Cancer Cell. 2009;15(3):232-239.

70. Paez-Ribes M, et al. Antiangiogenic therapy elicits malignant progression of tumors to increased local invasion and distant metastasis. Cancer Cell. 2009; 15(3):220-231.
71. Greenberg JI, et al. A role for VEGF as a negative regulator of pericyte function and vessel maturation. Nature. 2008;456(7223):809-813.

72. Stockmann C, et al. Deletion of vascular endothelial growth factor in myeloid cells accelerates tumorigenesis. Nature. 2008;456(7223):814-818.

73. Grulich AE, van Leeuwen MT, Falster MO, Vajdic $\mathrm{CM}$. Incidence of cancers in people with HIV/AIDS compared with immunosuppressed transplant recipients: a meta-analysis. Lancet. 2007;370(9581):59-67.

74. Shankaran V, et al. IFNgamma and lymphocytes prevent primary tumour development and shape tumour immunogenicity. Nature. 2001; 410(6832):1107-1111.

75. Lapidot $\mathrm{T}$, et al. A cell initiating human acute myeloid leukaemia after transplantation into SCID mice. Nature. 1994;367(6464):645-648.

76. Rosenberg SA, Restifo NP, Yang JC, Morgan RA, Dudley ME. Adoptive cell transfer: a clinical path to effective cancer immunotherapy. Nat Rev Cancer. 2008;8(4):299-308.

77. Medic S, Pearce RL, Heenan PJ, Ziman M. Molecular markers of circulating melanoma cells. Pigment Cell Res. 2007;20(2):80-91.

78. Juhasz I, et al. Growth and invasion of human melanomas in human skin grafted to immunodeficient mice. Am J Pathol. 1993;143(2):528-537.

79. Mapara MY, Sykes M. Tolerance and cancer: mechanisms of tumor evasion and strategies for breaking tolerance. J Clin Oncol. 2004;22(6):1136-1151.

80. Costello RT, et al. Human acute myeloid leukemia CD34+/CD38- progenitor cells have decreased sensitivity to chemotherapy and Fas-induced apoptosis, reduced immunogenicity, and impaired dendritic cell transformation capacities. Cancer Res. 2000; 60(16):4403-4411.

81. Kawasaki BT, Farrar WL. Cancer stem cells, CD200 and immunoevasion. Trends Immunol. 2008; 29(10):464-468.

82. Frank MH, Sayegh MH. Immunomodulatory functions of mesenchymal stem cells. Lancet. 2004; 363(9419):1411-1412.

83. Le Blanc K, et al. Treatment of severe acute graftversus-host disease with third party haploidentical mesenchymal stem cells. Lancet. 2004; 363(9419):1439-1441.

84. Le Blanc K, Ringden O. Immunomodulation by mesenchymal stem cells and clinical experience. J Intern Med. 2007;262(5):509-525.

85. Uccelli A, Moretta L, Pistoia V. Mesenchymal stem cells in health and disease. Nat Rev Immunol. 2008;8(9):726-736.

86. Gottesman MM, Fojo T, Bates SE. Multidrug resistance in cancer: role of ATP-dependent transporters. Nat Rev Cancer. 2002;2(1):48-58.

87. Dean M, Fojo T, Bates S. Tumour stem cells and drug resistance. Nat Rev Cancer. 2005;5(4):275-284.

88. de Grouw EP, et al. Preferential expression of a high number of ATP binding cassette transporters in both normal and leukemic CD34+CD38- cells. Lenkemia. 2006;20(4):750-754.

89. Ito $\mathrm{K}$, et al. PML targeting eradicates quiescent leukaemia-initiating cells. Nature. 2008; 453(7198):1072-1078.

90. Michor F, et al. Dynamics of chronic myeloid leukaemia. Nature. 2005;435(7046):1267-1270.

91. Viale A, et al. Cell-cycle restriction limits DNA damage and maintains self-renewal of leukaemia stem cells. Nature. 2009;457(7225):51-56.

92. Wulf GG, et al. A leukemic stem cell with intrinsic drug efflux capacity in acute myeloid leukemia. Blood. 2001;98(4):1166-1173.

93. Frank NY, et al. ABCB5-mediated doxorubicin transport and chemoresistance in human malignant melanoma. Cancer Res. 2005;65(10):4320-4333.

94. Eramo A, et al. Chemotherapy resistance of glioblastoma stem cells. Cell Death Differ. 2006; 13(7):1238-1241. 
95. Li X, et al. Intrinsic resistance of tumorigenic breast cancer cells to chemotherapy. J Natl Cancer Inst. 2008;100(9):672-679.

96. Yu F, et al. let-7 regulates self renewal and tumorigenicity of breast cancer cells. Cell. 2007; 131(6):1109-1123.

97. Dylla SJ, et al. Colorectal cancer stem cells are enriched in xenogeneic tumors following chemotherapy. PLoS One. 2008;3(6): 2428.

98. Bao S, et al. Glioma stem cells promote radioresistance by preferential activation of the DNA damage response. Nature. 2006;444(7120):756-760.

99. Diehn M, et al. Association of reactive oxygen species levels and radioresistance in cancer stem cells. Nature. 2009;458(7239):780-783.

100. Phillips TM, McBride WH, Pajonk F. The response of CD24(-/low)/CD44+ breast cancer-initiating cells to radiation. J Natl Cancer Inst. 2006;98(24):1777-1785.

101. Hoek KS, et al. In vivo switching of human melanoma cells between proliferative and invasive states. Cancer Res. 2008;68(3):650-656.

102. Hoek KS, Eichhoff OM, Widmer D, Dummer R Stemming the flood. Pigment Cell Melanoma Res. 2009;22(1):6-7.

103. Sousa JF, Espreafico EM. Suppression subtractive hybridization profiles of radial growth phase and metastatic melanoma cell lines reveal novel potential targets. BMC Cancer. 2008;8:19.

104.Klein WM, Wu BP, Zhao S, Wu H, Klein-Szanto AJ, Tahan SR. Increased expression of stem cell markers in malignant melanoma. Mod Pathol. 2007; 20(1):102-107.

105. Monzani E, et al. Melanoma contains CD133 and ABCG2 positive cells with enhanced tumourigenic potential. Eur J Cancer. 2007;43(5):935-946.

106. Rappa G, Fodstad O, Lorico A. The stem cell-associated antigen CD133 (Prominin-1) is a molecular therapeutic target for metastatic melanoma. Stem Cells. 2008;26(12):3008-3017.

107. Bao S, et al. Targeting cancer stem cells through L1CAM suppresses glioma growth. Cancer Res. 2008;68(15):6043-6048.

108. Guzman ML, et al. An orally bioavailable parthenolide analog selectively eradicates acute myelogenous leukemia stem and progenitor cells. Blood. 2007;110(13):4427-4435.

109. Jin L, et al. Monoclonal antibody-mediated targeting of CD123, IL-3 receptor alpha chain, eliminates human acute myeloid leukemic stem cells. Cell Stem Cell. 2009;5(1):31-42.

110.Yilmaz OH, et al. Pten dependence distinguishes haematopoietic stem cells from leukaemia-initiating cells. Nature. 2006;441(7092):475-482.

111.Elliott AM, Al-Hajj MA. ABCB8 mediates doxorubicin resistance in melanoma cells by protecting the mitochondrial genome. Mol Cancer Res. 2009; 7(1):79-87.

112.Huang Y, et al. Membrane transporters and channels: role of the transportome in cancer chemosensitivity and chemoresistance. Cancer Res. 2004 64(12):4294-4301.

113. Frank NY, Frank MH. ABCB5 gene amplification in human leukemia cells. Leuk Res. 2009; 33(10):1303-1305.

114.Lehne G, et al. Upregulation of stem cell genes in multidrug resistant K562 leukemia cells. Lenk Res. 2009;33(10):1379-1385.

115.Todaro $\mathrm{M}$, et al. Colon cancer stem cells dictate tumor growth and resist cell death by production of interleukin-4. Cell Stem Cell. 2007;1(4):389-402.

116.Vlashi E, McBride WH, Pajonk F. Radiation responses of cancer stem cells. J Cell Biochem. 2009; 108(2):339-342.

117. Chang CJ, et al. Enhanced radiosensitivity and radiation-induced apoptosis in glioma CD133-positive cells by knockdown of SirT1 expression. Biochem Biophys Res Commun. 2009;380(2):236-242.

118.Pierce GB. The cancer cell and its control by the embryo. Rous-Whipple Award lecture. Am J Pathol. 1983;113(1):117-124.

119.Lotem J, Sachs L. Epigenetics and the plasticity of differentiation in normal and cancer stem cells. Oncogene. 2006;25(59):7663-7672.

120.Jones PA, Baylin SB. The epigenomics of cancer. Cell. 2007;128(4):683-692.

121.Fan X, et al. Notch pathway inhibition depletes stem-like cells and blocks engraftment in embryonal brain tumors. Cancer Res. 2006;66(15):7445-7452.

122. Gupta PB, et al. Identification of selective inhibitors of cancer stem cells by high-throughput screening. Cell. 2009;138(4):645-659.

123.Jin L, Hope KJ, Zhai Q, Smadja-Joffe F, Dick JE. Targeting of CD44 eradicates human acute myeloid leukemic stem cells. Nat Med. 2006;12(10):1167-1174.

124.Arce C, et al. A proof-of-principle study of epigenetic therapy added to neoadjuvant doxorubicin cyclophosphamide for locally advanced breast cancer. PLoS One. 2006;1:e98.

125.Zhou BB, Zhang H, Damelin M, Geles KG, Grindley JC, Dirks PB. Tumour-initiating cells: challenges and opportunities for anticancer drug discovery. Nat Rev Drug Discov. 2009;8(10):806-823. 